\title{
molecules
}

ISSN 1420-3049

http://www.mdpi.org

\section{Synthesis of $N$-Phenylpyrrole Carboximides}

\author{
Dieter Hubmann ${ }^{1}$, Christoph Liechti ${ }^{1}$, Uwe Trinks ${ }^{2}$, Peter Traxler ${ }^{2}$ and Urs Séquin ${ }^{1} *$ \\ ${ }^{1}$ Institut für Organische Chemie der Universität Basel, St. Johanns-Ring 19. CH-4056 Basel, \\ Switzerland \\ Tel. +41 61 2671110, Fax +41 61 2671103, E-mail: sequin@ubaclu.unibas.ch \\ ${ }^{2}$ Novartis Pharma AG, CH-4002 Basel, Switzerland \\ *Author to whom correspondence should be addressed.
}

Received: 1 January 1999 / Accepted: 10 May 1999 / Published: 16 May 1999

\begin{abstract}
Several $N$-phenylpyrrole carboximides were synthesised using acyl isocyanates as intermediates.
\end{abstract}

Keywords: Acyl isocyanates, $N$-phenylpyrrole.

\section{Introduction}

During a certain stage of our synthetic approach towards potential protein tyrosine kinases inhibitors (see e. g. [1], [2]), compounds of the general structure 1 were required (Figure 1). Acyl isocyanates were used as intermediates for the synthesis of the imides of type 1. One route to this type of compounds consisted in the addition of a C-nucleophile to the isocyanate of a pyrrolecarboxylic acid, whereas in a different approach, an acyl isocyanate was used as the electrophile in a Friedel-Crafts type substitution of a suitable pyrrole derivative.

(C) 1999 by the authors. Reproduction of this article, by any means, is permitted for noncommercial purposes. 
<smiles>[R]C(=O)NC(=O)c1ccn([R])c1</smiles>

Figure 1. General structure of target compounds.

\section{Results and Discussion}

Reaction of $N$-phenylpyrrole-3-carboxylic acid (2) with thionyl chloride (Scheme 1) gave the acid chloride 3, which was treated with tetrabutylammonium isocyanate in tetrahydrofuran to yield the corresponding acyl isocyanate 4 . This sensitive compound could not be isolated, but its formation was easily demonstrated by quenching the reaction with ethanol, whereupon the acylated carbamate $\mathbf{5}$ was obtained. Reaction of $\mathbf{4}$ with lithium phenylacetylide gave the imide $\mathbf{6}$.

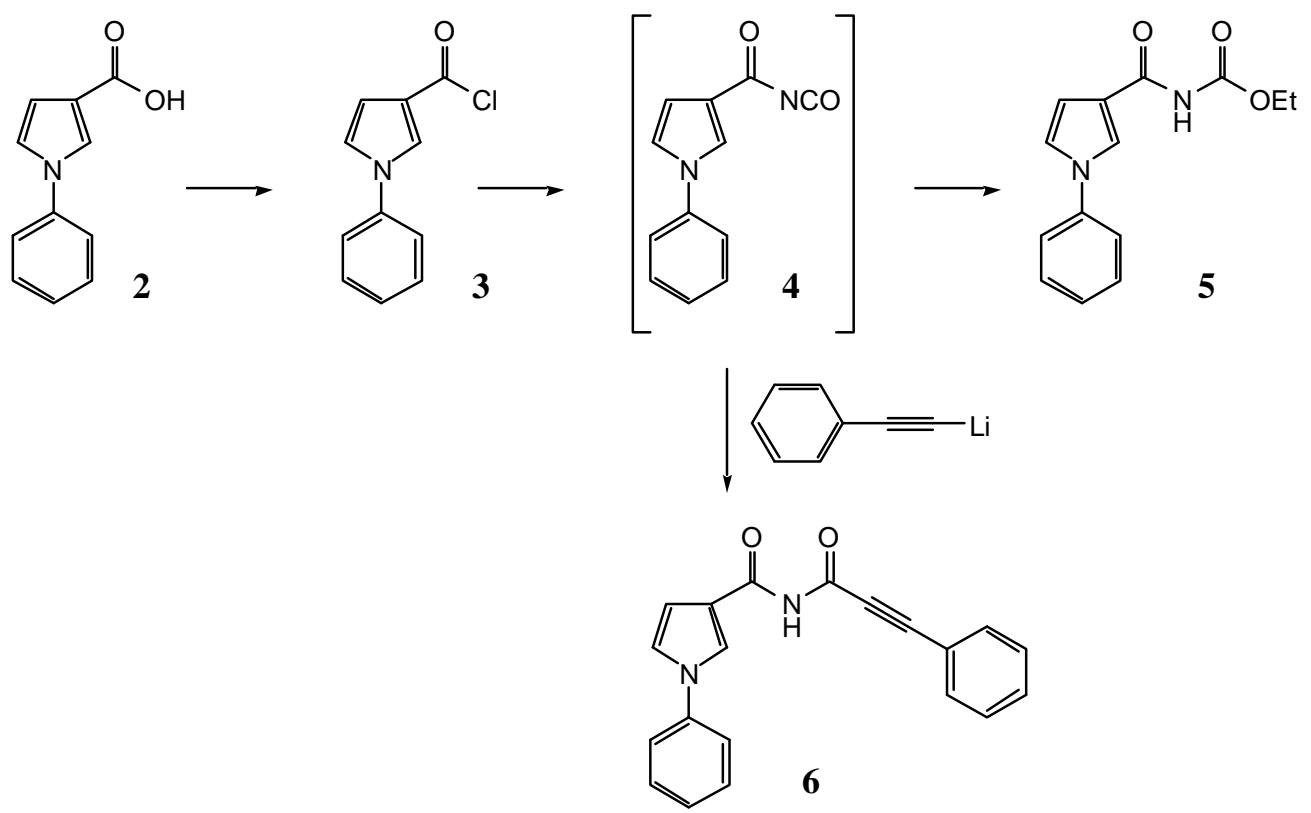

Scheme 1. Synthesis of imide 6.

Using the second approach mentioned, the isocyanate 7, which had been obtained from phenylpropiolamide and oxalyl chloride in dichloromethane, was reacted (Scheme 2) with the suitably protected $\mathrm{N}$-phenylpyrrole $\mathbf{8}$. Imide 9 was obtained in good yield; deprotection to $\mathbf{6}$, however, was difficult and could be achieved in only $23 \%$. When unprotected $N$-phenylpyrrole was treated with the isocyanate 7, substitution took place predominantly in the 2-position of the pyrrole ring, yielding $\mathbf{1 0}$. This latter compound cyclised to the oxazinone $\mathbf{1 1}$ upon heating. The structure of $\mathbf{1 1}$ was obtained from X-ray diffraction [3]. 


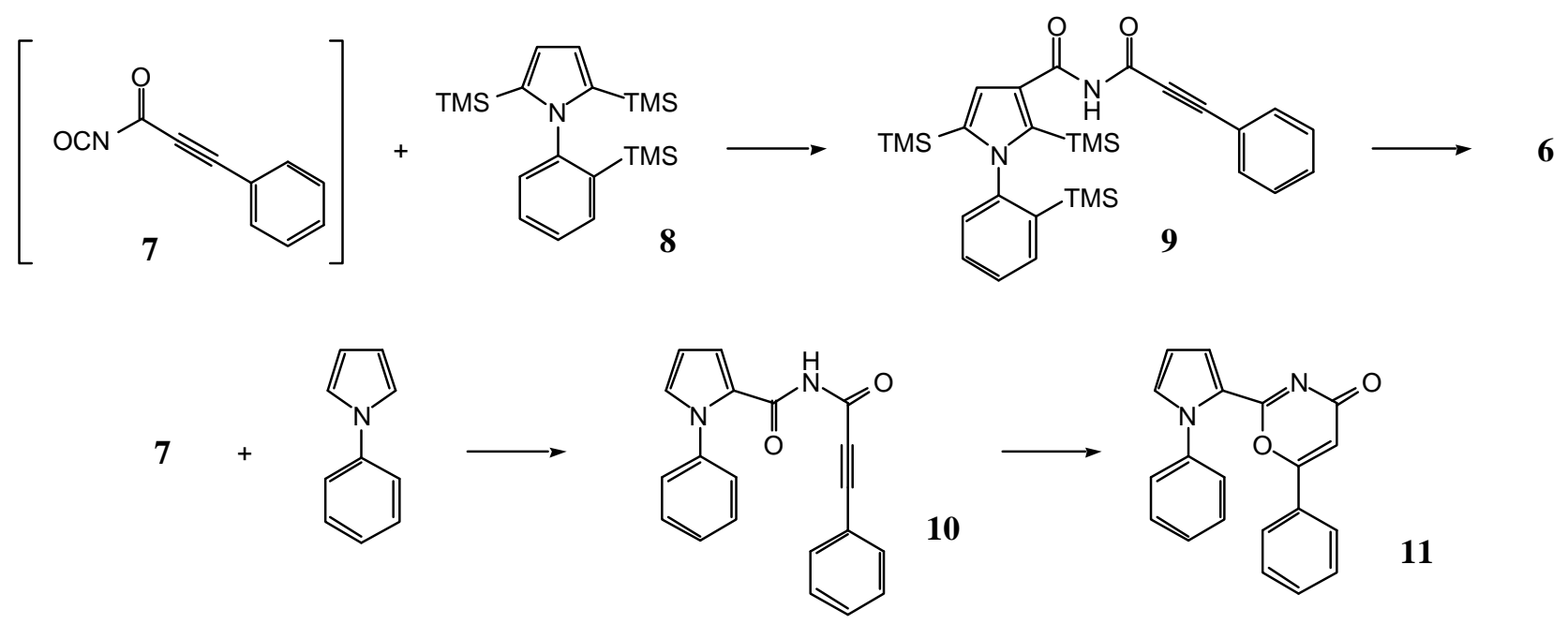

Scheme 2. Alternate synthesis of imide $\mathbf{6}$ and formation of oxazinone $\mathbf{1 1 .}$

\section{Experimental}

\section{General}

Chemicals were purchased from Fluka AG, Aldrich Chemical Company, Inc., Merck GmbH, or Lancaster Synthesis Ltd. Solvents used in reactions were distilled and dried or purchased in absolute quality. Tetrahydrofuran was freshly distilled from Na/K. TLC: Merck silica gel $60 \mathrm{~F}_{254}$ precoated glass plates. Column chromatography: flash-chromatography procedure of Still et al. [4]; columns with water cooling; Merck Kieselgel 60, 40-63 $\mu \mathrm{m}$.

M.p.: Kofler hot stage, corrected. IR: Perkin-Elmer FT-IR 1600; KBr pellet or liquid film between $\mathrm{NaCl}$ plates. NMR: Varian Gemini $300\left({ }^{1} \mathrm{H}: 300 \mathrm{MHz},{ }^{13} \mathrm{C}: 75 \mathrm{MHz}\right)$; chemical shifts $\delta$ in ppm relative to TMS (0 ppm); coupling constants $J$ in $\mathrm{Hz}$; multiplicities of ${ }^{13} \mathrm{C}$ resonances from APT and/or ${ }^{1} \mathrm{H},{ }^{13} \mathrm{C}$ COSY experiments; * means that similar assignments may be interchanged within the same spectrum. MS: VG 70-250 (Dr. H. Nadig); FAB MS: VG ZAB HF (courtesy Ciba-Geigy AG, Basel).

1-Phenyl-1H-pyrrole-3-carbonyl chloride (3)

A solution of 1-phenyl-1H-pyrrole-3-carboxylic acid [5] $(2,104 \mathrm{mg}, 556 \mu \mathrm{mol})$ and thionyl chloride (700 $\mu \mathrm{L}, 9.62 \mathrm{mmol})$ in hexane $(2 \mathrm{~mL})$ was stirred under Ar for $5 \mathrm{~h}$. The solvent was removed to give $126 \mathrm{mg}$ of 3 as a brownish oil (containing 10-20\% of the carboxylic acid), which was used without further purification [6].

Ethyl $N$-(1-phenyl-1H-pyrrole-3-carbonyl)carbamate (5)

Tetrabutylammonium isocyanate $(279 \mathrm{mg}, 981 \mu \mathrm{mol})$ was dried in vacuo at $65^{\circ}$ for $1 \mathrm{~h}$, disssolved 
under $\mathrm{Ar}$ in tetrahydrofuran $(1.7 \mathrm{~mL})$, and the solution cooled to $-78^{\circ}$. Ethanol (abs, $\left.50 \mu \mathrm{L}\right)$, and then a precooled solution of $\mathbf{3}(110 \mathrm{mg}, 535 \mu \mathrm{mol})$ were added under Ar. The mixture was stirred for $3 \mathrm{~h}$ and then the solvent removed in vacuo. The residue was chromatographed on $\mathrm{SiO}_{2}(27 \mathrm{~g}$, dichloromethane/ethyl acetate 8:1) to give $26 \mathrm{mg}$ (19\%) of 5 as a colorless solid, $20 \mathrm{mg}$ (20\%) of 1phenyl-1H-pyrrole-3-carboxamide, and $37 \mathrm{mg}$ of unidentified by-products.

Colorless solid, m.p. 44-46 .

IR (KBr): 3279; 3132; 2980; 2930; 1750; 1676; 1599; 1511; 1269; 1201; 1057; 1033; 901; 758; 692.

${ }^{1} \mathrm{H}$ NMR (300 MHz, $\left.\mathrm{CDCl}_{3}\right): 8.01(\mathrm{~s}, 1 \mathrm{H}, \mathrm{NH}) ; 7.78(\mathrm{t}, J=2.0,1 \mathrm{H}, \mathrm{H}-\mathrm{C}(2)) ; 7.5-7.3(\mathrm{~m}, 5 \mathrm{H}$, phenyl-H); 7.05 (dd, $J=2.3,3.1,1 \mathrm{H}, \mathrm{H}-\mathrm{C}(5)) ; 6.67$ (dd, $J=3.0,1.7,1 \mathrm{H}, \mathrm{H}-\mathrm{C}(4)) ; 4.30$ (q, $J=7.1$, $\left.2 \mathrm{H}, \mathrm{CH}_{2}\right) ; 1.33\left(\mathrm{t}, J=6.9,3 \mathrm{H}, \mathrm{CH}_{3}\right)$.

${ }^{13} \mathrm{C}$ NMR (75 MHz, $\left.\mathrm{CDCl}_{3}\right)$ : $161.1(\mathrm{C}=\mathrm{O}) ; 151.4(\mathrm{O}-\mathrm{C}=\mathrm{O}) ; 139.5$ (phenyl C(1)); 129.8 (phenyl $\mathrm{C}(3), \mathrm{C}(5)) ; 127.2$ (phenyl C(4)); 124.0 (C(2)); 121.1 (C(5)); 121.0 (phenyl C(2), C(6)); 120.2 (C(3)); $109.5(\mathrm{C}(4)) ; 62.1\left(\mathrm{CH}_{2}\right) ; 14.3\left(\mathrm{CH}_{3}\right)$.

EI MS: 259 (3); $258\left(20, M^{+}\right) ; 212$ (13); $171(13) ; 170\left(100,\left[M-\mathrm{NH}-\mathrm{COOC}_{2} \mathrm{H}_{5}\right]^{+}\right) ; 115$ (13); 77 (17); 51 (12).

Anal. Calcd for $\mathrm{C}_{14} \mathrm{H}_{14} \mathrm{~N}_{2} \mathrm{O}_{3}$ (258.28): C, 65.11; H, 5.46; N, 10.85; O, 18.58. Found: C, 64.91; H, 5.67; N, 10.04; O, 17.11.

$N$-(3-Phenyl-2-propynoyl)-1-phenyl-1H-pyrrole-3-carboxamide $\quad(6) \quad$ from 1 -phenyl-1H-pyrrole-3carbonyl chloride (3)

To a solution of tetrabutylammonium cyanate $(909 \mathrm{mg}, 3.20 \mathrm{mmol})$ in tetrahydrofuran $(12 \mathrm{~mL}), 1$ phenyl-1H-pyrrole-3-carbonyl chloride (3, $465 \mathrm{mg}, 2.26 \mathrm{mmol})$ was added under Ar. After stirring the mixture for $90 \mathrm{~min}$ at $0^{\circ}$, lithium phenylacetylide $(2.26 \mathrm{ml}, 1.0 \mathrm{M}$ in tetrahydrofuran, $2.26 \mathrm{mmol}$ ) was added and the mixture stirred for additional $3 \mathrm{~h}$ at $0^{\circ}$. The solvent was removed in vacuo and the residue chromatographed on $\mathrm{SiO}_{2}$ (100 g, dichloromethane) to give $104 \mathrm{mg}$ (15\%) of 6 as a colorless solid.

Colorless needles (dichloromethane/pentane), m.p. 149-151 ${ }^{\circ}$.

IR (KBr): 3251; 3151; 2230; 2196; 1709, 1637; 1336; 1250; 754; 747.

${ }^{1} \mathrm{H}$ NMR $\left(300 \mathrm{MHz}, \mathrm{CDCl}_{3}\right): 9.79(s b r, 1 \mathrm{H}, \mathrm{NH}) ; 8.03(t, J=2.0,1 \mathrm{H}, \mathrm{H}-\mathrm{C}(2)) ; 7.67(d d, J=6.8$, $1.5,2 \mathrm{H}, \mathrm{H}-\mathrm{C}(2), \mathrm{C} \equiv \mathrm{C}-$ phenyl H-C(6)); 7.45-7.35 ( $m, 8 \mathrm{H}$, phenyl-H); $7.10(t, J=3.1,1 \mathrm{H}, \mathrm{H}-\mathrm{C}(5))$; $6.89(d d, J=3.1,1.8,1 \mathrm{H}, \mathrm{H}-\mathrm{C}(4))$.

${ }^{13} \mathrm{C}$ NMR $\left(75 \mathrm{MHz}, \mathrm{CDCl}_{3}\right): 161.2,153.8(2 \times \mathrm{C}=\mathrm{O}) ; 139.5(N$-phenyl $\mathrm{C}(1)) ; 133.1(\mathrm{C} \equiv \mathrm{C}-$ phenyl $\mathrm{C}(2), \mathrm{C}(6))$; 130.7 (C $\equiv \mathrm{C}$-phenyl $\mathrm{C}(4)) ; 129.8,128.5$ ( $N$-phenyl $\mathrm{C}(3), \mathrm{C}(5)$, and $\mathrm{C} \equiv \mathrm{C}$-phenyl $\mathrm{C}(3)$, $\mathrm{C}(5))$; 127.1 (N-phenyl C(4)); $124.4\left(\mathrm{C}(2)^{*}\right) ; 121.3\left(\mathrm{C}(5)^{*}\right) ; 120.8(N$-phenyl C(2), C(6)); $120.1(\mathrm{C} \equiv \mathrm{C}$ phenyl $\left.\mathrm{C}(1)^{*}\right) ; 119.6\left(\mathrm{C}(3)^{*}\right) ; 110.5(\mathrm{C}(4)) ; 93.5(C \equiv \mathrm{C}-\mathrm{CO}) ; 83.3(\mathrm{C} \equiv C-\mathrm{CO})$.

EI MS (70 eV): $314\left(7, M^{+}\right) ; 168$ (100); $140(5) ; 118(24) ; 105$ (10); 90 (7); $77\left(15,\left[\mathrm{C}_{6} \mathrm{H}_{5}\right]^{+}\right) ; 51$ 
(7).

CI MS $\left(\mathrm{NH}_{3}\right): 315\left(100,[M+\mathrm{H}]^{+}\right) ; 186\left(11,\left[\mathrm{C}_{6} \mathrm{H}_{5}-\mathrm{C}_{4} \mathrm{H}_{3} \mathrm{~N}-\mathrm{CONH}_{2}\right]^{+}\right) ; 164$ (5).

EI HRMS (70 eV): Calcd for $\mathrm{C}_{20} \mathrm{H}_{14} \mathrm{~N}_{2} \mathrm{O}_{2}$ : 314.1055. Found: 314.1049 .

2,5-Bis(trimethylsilyl)-1-[2-(trimethylsilyl)phenyl]-1H-pyrrole (8)

Butyllithium (100 mL, $1.6 \mathrm{M}$ in hexane, $160.0 \mathrm{mmol}$ ) was added under Ar to 1-phenyl-1H-pyrrole (5.09 g, $36.0 \mathrm{mmol})$ and $N, N, N^{\prime}, N^{\prime}$-tetramethylethylenediamine $(22.5 \mathrm{~mL}, 160.0 \mathrm{mmol})$. The mixture was refluxed for $23 \mathrm{~h}$ and then cooled to $-78^{\circ}$. Trimethylchlorosilane $(20.0 \mathrm{~mL}, 160.0 \mathrm{mmol})$ was added and the mixture stirred for $6 \mathrm{~h}$ at $0^{\circ}$ and for $90 \mathrm{~min}$ at room temperature. After washing twice with sat. $\mathrm{NH}_{4} \mathrm{Cl}$ solution and then with water, the organic layer was dried $\left(\mathrm{Na}_{2} \mathrm{SO}_{4}\right)$, filtered, and the solvent evaporated. The crude product ( $12.4 \mathrm{~g}$ of a yellowish oil) was purified in ten portions by chromatography on $\mathrm{SiO}_{2}(150 \mathrm{~g}$, pentane/dichloromethane $12: 1)$ to give $4.85 \mathrm{~g}(37 \%)$ of $\mathbf{8}$ as a yellowish oil. An analytically pure sample was obtained by kugelrohr distillation (175\% $0.13 \mathrm{mbar})$.

IR (NaCl): 3060; 2956; 2897; 1479; 1247; 1166; 1120; 931; 837; 757.

${ }^{1} \mathrm{H}$ NMR $\left(300 \mathrm{MHz}, \mathrm{CDCl}_{3}\right): 7.54(d d, J=7.3,1.8,1 \mathrm{H}$, phenyl H-C(3)); $7.38(t d, J=7.4,1.5,1 \mathrm{H}$, phenyl H-C(4)*); $7.31\left(t d, J=7.4,1.7,1 \mathrm{H}\right.$, phenyl $\left.\mathrm{H}-\mathrm{C}(5)^{*}\right) ; 7.15(d d, J=7.7,1.2,1 \mathrm{H}$, phenyl H-C(6)); $6.48(s, 2 \mathrm{H}, \mathrm{H}-\mathrm{C}(3), \mathrm{H}-\mathrm{C}(4)) ;-0.01\left(s, 9 \mathrm{H},\left(\mathrm{CH}_{3}\right)_{3} \mathrm{Si}\right.$-phenyl); $-0.09\left(s, 18 \mathrm{H},\left(\mathrm{CH}_{3}\right)_{3} \mathrm{Si}\right.$-pyrrole $)$.

${ }^{13} \mathrm{C}$ NMR (75 MHz, $\left.\mathrm{CDCl}_{3}\right): 147.9$ ( $s$, phenyl C(1)); $140.2(s$, phenyl $\mathrm{C}(2)) ; 140.0(s, \mathrm{C}(2), \mathrm{C}(5))$; 135.2 (d, phenyl C(3); 130.0, 128.4, 127.8 (3d, phenyl C(4), C(5), C(6)); 119.1 (d, C(3), C(4)); $0.3(q$, $\left(\mathrm{CH}_{3}\right)_{3} \mathrm{Si}$-pyrrole); $-0.5\left(q,\left(\mathrm{CH}_{3}\right)_{3} \mathrm{Si}\right.$-phenyl).

EI MS (70 eV): $359\left(10, M^{+}\right) ; 286\left(24,\left[M-\mathrm{Si}\left(\mathrm{CH}_{3}\right)_{3}\right]^{+}\right) ; 256$ (23); 240 (5); 212 (9); 198 (14); 73 $\left(100,\left[\mathrm{Si}\left(\mathrm{CH}_{3}\right)_{3}\right]^{+}\right)$.

CI MS $\left(\mathrm{NH}_{3}\right): 360\left(100,[M+\mathrm{H}]^{+}\right) ; 288\left(21,\left[M-\mathrm{Si}\left(\mathrm{CH}_{3}\right)_{3}+2 \mathrm{H}\right]^{+}\right) ; 214$ (7); 90 (30); 73 (6, $\left.\left[\mathrm{Si}\left(\mathrm{CH}_{3}\right)_{3}\right]^{+}\right)$.

$N$-(3-Phenyl-2-propynoyl)-2,5-bis(trimethylsilyl)-1-[2-(trimethylsilyl)phenyl]-1 $H$-pyrrole-3-carboxamide (9)

To a solution of 3-phenyl-2-propynamide [7] (741 mg, $5.10 \mathrm{mmol}$, prepared from 3-phenyl-2propynoyl chloride [8]) in dichloromethane $(12 \mathrm{~mL})$, oxalyl chloride $(482 \mu \mathrm{L}, 5.62 \mathrm{mmol})$ was added under $\mathrm{Ar}$ at room temperature. The mixture was refluxed for $135 \mathrm{~min}$ and then cooled to $0^{\circ}$. 2,5Bis(trimethylsilyl)-1-[2-(trimethylsilyl)phenyl]-1H-pyrrole $(\mathbf{8}, 820 \mu \mathrm{L}, 2.10 \mathrm{mmol})$ in dichloromethane $(5 \mathrm{~mL})$ was added under $\mathrm{Ar}$, followed by $\mathrm{AlCl}_{3}(1.472 \mathrm{~g}, 11.04 \mathrm{mmol})$. The mixture was stirred for $22 \mathrm{~h}$ at $0^{\circ}$ and then poured into ice/water $(100 \mathrm{~mL})$. After stirring for $2.5 \mathrm{~h}$, the organic phase was separated, dried $\left(\mathrm{Na}_{2} \mathrm{SO}_{4}\right)$, filtered, and the solvent evaporated in vacuo. The residue was chromatographed on $\mathrm{SiO}_{2}$ (95g, gradient dichloromethane $\rightarrow$ dichloromethane/methanol 99:1) to give $852 \mathrm{mg}(76 \%)$ of 9 as a colorless solid. 
Colorless prisms (tert-butyl methyl ether/pentane), m.p. 175-177 ${ }^{\circ}$.

IR (KBr): 3272; 3059; 2954; 2896; 2234; 2199; 1703; 1634; 1336; 1215; 838; 760.

${ }^{1} \mathrm{H}$ NMR (300 MHz, $\left.\mathrm{CDCl}_{3}\right): 8.87(s \mathrm{br}, 1 \mathrm{H}, \mathrm{NH}) ; 7.66(d t, J=6.7,1.6,2 \mathrm{H}, \mathrm{C} \equiv \mathrm{C}-$ phenyl H-C(2), $\mathrm{H}-\mathrm{C}(6)) ; 7.57(d d, J=7.4,1.5,1 \mathrm{H}, N$-phenyl H-C(3)); 7.47-7.33 ( $m, 5 \mathrm{H}$, phenyl-H); $7.12(d d, J=7.6$, $1.5,1 \mathrm{H}, N$-phenyl H-C(6)); $6.78(s, 1 \mathrm{H}, \mathrm{H}-\mathrm{C}(4)) ; 0.02,-0.01,-0.07\left(3 s, 3 \times 9 \mathrm{H}, 3 \times\left(\mathrm{CH}_{3}\right)_{3} \mathrm{Si}\right)$.

${ }^{13} \mathrm{C}$ NMR $\left(75 \mathrm{MHz}, \mathrm{CDCl}_{3}\right): 161.7 s, 152.8 s(2 \times \mathrm{C}=\mathrm{O}) ; 147.0 s ; 146.9 s ; 141.5 s ; 139.9 s ; 135.4 d$; $133.2 d ; 130.6 d ; 129.7 d ; 128.8 d ; 128.6 d ; 128.5 d ; 126.3 s ; 120.4 s ; 118.8 d ; 91.8 s$ (C三C-CO); $83.2 s$ $(\mathrm{C} \equiv C-\mathrm{CO}) ; 0.1 q,-0.1 q-0.7 q\left(3 \times\left(\mathrm{CH}_{3}\right)_{3} \mathrm{Si}\right)$.

EI MS (70 eV): $530\left(6, M^{+}\right) ; 515\left(9,\left[M-\mathrm{CH}_{3}\right]^{+}\right) ; 457\left(7,\left[M-\mathrm{Si}\left(\mathrm{CH}_{3}\right)_{3}\right]^{+}\right) ; 384(28,[M-$ $\left.\left.2 \times \mathrm{Si}\left(\mathrm{CH}_{3}\right)\right]^{+}\right) ; 311\left(23,\left[M-3 \times \mathrm{Si}\left(\mathrm{CH}_{3}\right)_{3}\right]^{+}\right) ; 129$ (17); 118 (9); 89 (5); $77\left(5,\left[\mathrm{C}_{6} \mathrm{H}_{5}\right]^{+}\right) ; 73(100$, $\left.\left[\mathrm{Si}\left(\mathrm{CH}_{3}\right)_{3}\right]^{+}\right) ; 45(15)$.

Anal. Calcd for $\mathrm{C}_{29} \mathrm{H}_{38} \mathrm{~N}_{2} \mathrm{O}_{2} \mathrm{Si}_{3}$ (530.89): C, 65.61; H, 7.22; N, 5.28. Found: C, 65.72; H, 7.30; N, 5.10 .

$N$-(3-Phenyl-2-propynoyl)-1-phenyl-1H-pyrrole-3-carboxamide (6) from (9)

$N$-(3-Phenyl-2-propynoyl)-2,5-bis(trimethylsilyl)-1-[2-(trimethylsilyl)phenyl]-1H-pyrrole-3-

carboxamide $(9,87 \mathrm{mg}, 164 \mu \mathrm{mol})$ and tetrabutylammonium fluoride trihydrate $(218 \mathrm{mg}, 691 \mu \mathrm{mol})$ were stirred in tetrahydrofuran for $50 \mathrm{~min}$ at $60^{\circ}$. The mixture was then taken up with dichloromethane and washed with water. The organic phase was separated, dried over $\mathrm{Na}_{2} \mathrm{SO}_{4}$, filtered, and the solvent removed in vacuo. The residue was chromatographed on $\mathrm{SiO}_{2}$ (15 g, dichloromethane/methanol 99:1) to give $12 \mathrm{mg}(23 \%)$ of $\mathbf{6}$ as a colorless solid.

$N$-(3-Phenyl-2-propynoyl)-1-phenyl-1H-pyrrole-2-carboxamide (10) and 1,2-bis-(1-phenyl-1H-pyrrol-2yl)-ethane-1,2-dione (12)

To a solution of 3-phenyl-2-propynamide [7] (812 mg, $5.59 \mathrm{mmol})$ in dichloromethane (12 $\mathrm{mL})$, oxalyl chloride $(527 \mu \mathrm{L}, 6.15 \mathrm{mmol})$ was added under Ar at room temperature. The mixture was refluxed for $150 \mathrm{~min}$. 1-Phenyl- $1 H$-pyrrole $(820 \mathrm{mg}, 5.73 \mathrm{mmol})$ in dichloromethane $(3 \mathrm{~mL})$ was added and the mixture refluxed for $45 \mathrm{~h}$. The solvent was removed and the residue chromatographed on $\mathrm{SiO}_{2}$ (100 g, gradient dichloromethane $\rightarrow$ dichloromethane/methanol 99:1 $\rightarrow$ dichloromethane/methanol 98:2) to give $813 \mathrm{mg}(46 \%)$ of $\mathbf{1 0 .}$

Colorless needles (dichloromethane/pentane), mp. 125-127 ${ }^{\circ}$.

IR (KBr): 3215; 3125; 2211; 1702; 1654; 1598; 1498; 1261; 1187; 1170; 754; 744; 694.

${ }^{1} \mathrm{H}$ NMR $\left(300 \mathrm{MHz}, \mathrm{CDCl}_{3}\right): 8.74(s b r, 1 \mathrm{H}, \mathrm{NH}) ; 7.55(d d, J=6.9,1.5,2 \mathrm{H}, \mathrm{C} \equiv \mathrm{C}-$ phenyl H-C(2), $\mathrm{H}-\mathrm{C}(6)) ; 7.47-7.30(m, 8 \mathrm{H}$, phenyl-H); $7.06(d d, J=4.0,1.7,1 \mathrm{H}, \mathrm{H}-\mathrm{C}(3)) ; 7.04(d d, J=2.7,1.7,1 \mathrm{H}$, $\mathrm{H}-\mathrm{C}(5)) ; 6.35(d d, J=4.0,2.7,1 \mathrm{H}, \mathrm{H}-\mathrm{C}(4))$.

${ }^{13} \mathrm{C}$ NMR $\left(75 \mathrm{MHz}, \mathrm{CDCl}_{3}\right): 157.0 s, 152.9 s(2 \times \mathrm{C}=\mathrm{O}) ; 139.8 s(N$-phenyl $\mathrm{C}(1)) ; 132.9 d(\mathrm{C} \equiv \mathrm{C}-$ 


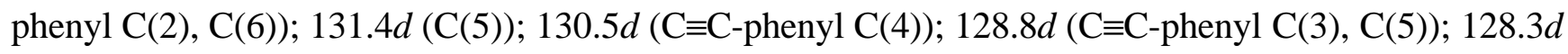
$(N$-phenyl C(3), C(5)); 127.8d ( $N$-phenyl C(4)); 125.8d ( $N$-phenyl C(2), C(6)); $124.4 s$ ( C(2)); $119.8 s$ $(\mathrm{C} \equiv \mathrm{C}$-phenyl $\mathrm{C}(1))$; $118.3 d(\mathrm{C}(3)) ; 109.7 d(\mathrm{C}(4))$; $92.4 s \quad(C \equiv \mathrm{C}-\mathrm{CO}) ; 82.9 s \quad(\mathrm{C} \equiv C-\mathrm{CO})$; assignments from ${ }^{1} \mathrm{H},{ }^{13} \mathrm{C}$ COSY.

EI MS (70 eV): $314\left(25, M^{+}\right) ; 291$ (13); 168 (100); 140 (12); 118 (55); 105 (28); 90 (16); 77 (36, $\left.\left[\mathrm{C}_{6} \mathrm{H}_{5}\right]^{+}\right) ; 51(22)$.

Anal. Calcd for $\mathrm{C}_{20} \mathrm{H}_{14} \mathrm{~N}_{2} \mathrm{O}_{2}$ (314.34): C, 76.42; H, 4.49; N, 8.91; O, 10.18. Found: C, 76.33; H, $4.48 ; \mathrm{N}, 8.85 ; \mathrm{O}, 10.23$.

As a by-product, 1,2-bis-(1-phenyl-1H-pyrrol-2-yl)-ethane-1,2-dione (12, $168 \mathrm{mg}, 9 \%)$ was isolated.

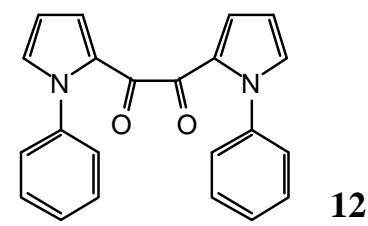

Figure 2.

Yellow leaflets (dichloromethane/pentane), m.p. 127.5-130.5 .

IR (KBr): 3127, 3064; 1644; 1629; 1495; 1407; 1351; 777; 753; 733; 698.

${ }^{1} \mathrm{H}$ NMR (300 MHz, $\left.\mathrm{CDCl}_{3}\right)$ : 7.41-7.27 ( $m, 10 \mathrm{H}$, phenyl-H); $7.10(d d, J=4.1,1.7,2 \mathrm{H}$, pyrrole H$\mathrm{C}(3)) ; 7.03(d d, J=2.5,1.7,2 \mathrm{H}$, pyrrole $\mathrm{H}-\mathrm{C}(5)) ; 6.28(d d, J=4.1,2.5,2 \mathrm{H}$, pyrrole $\mathrm{H}-\mathrm{C}(4))$.

${ }^{13} \mathrm{C}$ NMR (75 MHz, $\mathrm{CDCl}_{3}$ ): $181.2(\mathrm{C}=\mathrm{O}) ; 139.8$ (phenyl $\mathrm{C}(1)$ ); 132.9 (pyrrole $\left.\mathrm{C}(3)^{*}\right) ; 128.6$ (phenyl C(3'), $\mathrm{C}(5)$ ); 128.2 (pyrrole $\mathrm{C}(2)$ ); 127.8 (phenyl $\mathrm{C}\left(4^{\prime}\right)$ ); 125.7 (phenyl C(2), C(6)); 124.9 (pyrrole $\left.\mathrm{C}(5)^{*}\right) ; 110.4$ (pyrrole $\mathrm{C}\left(4^{\prime}\right)$ ).

EI MS (70 eV): $\left.340\left(7, M^{+}\right) ; 170\left(100,\left[\mathrm{C}_{6} \mathrm{H}_{5} \mathrm{C}_{4} \mathrm{H}_{3} \mathrm{NCO}\right]^{+}\right) ; 115(31) ; 77\left(5, \mathrm{C}_{6} \mathrm{H}_{5}\right]^{+}\right)$.

Anal. Calcd for $\mathrm{C}_{20} \mathrm{H}_{16} \mathrm{~N}_{2} \mathrm{O}_{2}$ (340.38): C, 77.63; H, 4.74; N, 8.23. Found: C, 77.15; H, 4.73; N, 8.19 .

6-Phenyl-2-(1-phenyl-1H-pyrrol-2-yl)-[1,3]oxazin-4-one (11)

$N$-(3-phenyl-2-propynoyl)-1-phenyl-1H-pyrrole-2-carboxamide (10, $33 \mathrm{mg}, 105 \mathrm{mmol})$ was heated under Ar to $160^{\circ}$ for $25 \mathrm{~min}$. After cooling, the brownish oil was chromatographed on $\mathrm{SiO}_{2}(24 \mathrm{~g}$, dichloromethane/methanol 99:1) to give $22 \mathrm{mg}$ (67\%) of $\mathbf{1 1}$ as a brownish solid.

Yellowish prisms (hexane/dichloromethane), m.p. 174-176 ${ }^{\circ}$.

IR (KBr): 3075; 2922; 1671, 1640; 1553; 1495; 1448; 1348; 948; 767; 734; 698.

${ }^{1} \mathrm{H}$ NMR $\left(300 \mathrm{MHz}, \mathrm{CDCl}_{3}\right): 7.56(d d, J=4.0,1.8,1 \mathrm{H}$, pyrrole $\mathrm{H}-\mathrm{C}(3)) ; 7.49-7.39(m, 6 \mathrm{H}, \mathrm{N}-$ phenyl-H and C=C-phenyl H-C(4)); 7.26 ( $t, J=8,2 \mathrm{H}, \mathrm{C}=\mathrm{C}$-phenyl $\mathrm{H}-\mathrm{C}(3), \mathrm{H}-\mathrm{C}(5)) ; 7.09$ (dd, $J=2.6$, 1.8, 1H, pyrrole $\mathrm{H}-\mathrm{C}(5))$; 6.94-6.90 ( $m, 2 \mathrm{H}, \mathrm{C}=\mathrm{C}-$ phenyl $\mathrm{H}-\mathrm{C}(2), \mathrm{H}-\mathrm{C}(6)) ; 6.48(s, 1 \mathrm{H}, \mathrm{H}-\mathrm{C}(5)) ; 6.45$ 
$(d d, J=4.0,2.6,1 \mathrm{H}$, pyrrole $\mathrm{H}-\mathrm{C}(4))$.

${ }^{13} \mathrm{C}$ NMR (75 MHz, $\left.\mathrm{CDCl}_{3}\right)$ : 168.1s (C(4)); 162.2s, 158.8s (C(2), C(6)); 140.7s ( $N$-phenyl C(1)); 132.2d (pyrrole $\mathrm{C}(5)) ; 131.7 d(\mathrm{C}=\mathrm{C}$-phenyl $\mathrm{C}(4)) ; 129.2 s$ (C=C-phenyl $\mathrm{C}(1)) ; 128.2 d$ ( $N$-phenyl $\mathrm{C}(4))$; $129.5 d, 128.8 d, 126.0 d, 125.3 d$ (C=C-phenyl and $N$-phenyl C(3), $\mathrm{C}(5), \mathrm{C}(2), \mathrm{C}(6)$ ); 123.1s (pyrrole $\mathrm{C}(2)$ ); $122.3 d$ (pyrrole $\mathrm{C}(3)$ ); $111.0 d$ (pyrrole $\mathrm{C}(4)$ ); $103.8 d\left(\mathrm{C}(5)\right.$ ); assignments from ${ }^{1} \mathrm{H},{ }^{13} \mathrm{C}$ COSY.

EI MS (70 eV): $314\left(22, M^{+}\right) ; 168(100) ; 140(7) ; 115(10) ; 77\left(19,\left[\mathrm{C}_{6} \mathrm{H}_{5}\right]^{+}\right)$.

Anal. Calcd for $\mathrm{C}_{20} \mathrm{H}_{14} \mathrm{~N}_{2} \mathrm{O}_{2}$ (314.34): C, 76.42; H, 4.49; N, 8.91; O, 10.18. Found: C, 76.42; H, $4.62 ; \mathrm{N}, 8.64 ; \mathrm{O}, 10.22$.

Crystals obtained from ethanol/water were subjected to X-ray structure determination [3].

Acknowledgements: Financial support by the Schweizerischer Nationalfonds zur Förderung der wissenschaftlichen Forschung (project no. 20-41857.94) is gratefully acknowledged.

\section{References and Notes}

1. Traxler, P.; Lydon, N. Recent advances in protein tyrosine kinase inhibitors. Drugs Fut. 1995, 20, 1261-1274.

2. Traxler, P.; Green, J.; Mett, H.; Séquin, U.; Furet, P. Use of a Pharmacophore Model for the Design of EGFR Tyrosine Kinase Inhibitors: Isoflavones and 3-Phenyl-4(1H)-qionolones. J. Med. Chem. 1999, 42, 1018-1026.

3. Hubmann, D. Zur Synthese von 2,8-Diphenylpyrrolo[3,4-c]azepin-4,6-dion, einem potentiellen Inhibitor der Epidermal Growth Factor Receptor Protein-Tyrosin-Kinase. Dissertation, Universität Basel, 1997.

4. Still, W. C.; Kahn, M.; Mitra, A. Rapid Chromatographic Technique for Preparative Separations with Moderate Resolution. J. Org. Chem. 1978, 43, 2923-2925.

5. Fabis, F.; Dallemagne, P.; Rault, S.; Robba, M. A new efficient synthesis of 3-amino-1phenylpyrrole. Org. Prep. Proced. Int. 1995, 27, 236-239.

6. Liechti, Ch. Synthese von Epidermal Growth Factor-Rezeptor-Protein-Tyrosin-Kinase-Inhibitoren. Diplomarbeit, Universität Basel, Olten 1994.

7. Rinkes, I. J. De l'action de l'hypochlorite de sodium sur les amides d'acides. Recl. Trav. Chim. Pays-Bas 1920, 39, 704-710.

8. Bergmann, F.; Haskelberg, L. Synthesis of Lipophilic Chemotherapeuticals. V. N ${ }^{4}$-Acylsulfanilamides. J. Am. Chem. Soc. 1941, 63, 2243-2245.

Sample Availability: Available from the authors.

(C) 1999 by the authors. Reproduction of this article, by any means, is permitted for noncommercial purposes. 